\title{
Numerical Observation of the Rescattering Wave Packet in Laser-Atom Interactions
}

\author{
X. M. Tong, ${ }^{1,2, *}$ S. Watahiki, ${ }^{1}$ K. Hino, ${ }^{1}$ and N. Toshima ${ }^{1}$ \\ ${ }^{1}$ Institute of Materials Science, Graduate School of Pure and Applied Sciences, University of Tsukuba, 1-1-1 Tennodai, \\ Tsukuba, Ibaraki 305-8573, Japan \\ ${ }^{2}$ Center for Computational Sciences, University of Tsukuba, 1-1-1 Tennodai, Tsukuba, Ibaraki 305-8577, Japan
}

(Received 12 April 2007; published 28 August 2007)

\begin{abstract}
We present a full-quantum nonperturbative method to study the electron rescattering process in the intense laser-atom interactions. We separate the ionized wave function from the background by solving the time-integral equation. Imposing the incoming boundary condition on the wave function, we reproduce the motion of the rescattering wave packet predicted by the rescattering theory. Our calculated rescattering energies differ significantly from the semiclassical ones. The difference would be substantial for the evaluation of the rescattering induced dynamics such as the molecular dissociation.
\end{abstract}

PACS numbers: $32.80 . \mathrm{Fb}, 32.80 . \mathrm{Wr}, 34.80 . \mathrm{Dp}, 42.65 . \mathrm{Re}$

Intense lasers interacting with atoms, molecules, and solid materials has become one of the hot research topics owing to the advances of laser technology. When the interaction strength of an electron with a laser field is comparable to the electron-core interaction, nonlinear processes could happen, such as the high-order harmonic generation (HHG) and the above threshold ionization (ATI). A key idea to understanding HHG and ATI is the rescattering theory of Corkum [1] based on a three-step model. First, the electron is ionized by the laser field through tunneling ionization when the laser field reaches its peak strength. Then, the ionized electron is bounced back by the laser field when it changes the direction (rescattering process). Finally, the rescattering electron collides with the parent core. This process is similar to the conventional electron beam incidents on the target. Differing from the conventional electron beam, the rescattering electron provides a high intensity coherent electron beam source. Most of the dynamic processes of the electron colliding with the target can be enhanced with this new electron beam source. For example, the returning electron can emit light due to HHG by the radiative recombination with the parent core, which opens a way to create the $\mathrm{x}$-ray laser. Electrons colliding with the parent core may induce other processes, e.g., the nonsequential double ionization [2], and the rescattering induced dissociation [3]. The rescattering wave packet can be used to generate a single attosecond pulse [4] or pulse trains [5]. It can also be used to image the molecular structure $[6,7]$. Thus, the quantitative description of the rescattering electron is highly desired.

There are two well-known models to study the rescattering induced processes. One is the classical model based on the rescattering theory in which the electron-core Coulomb interaction is ignored. The highest rescattering energy from this mode is in reasonable agreement with the observed HHG cutoff position [1]. However, the model fails to explain the nonsequential double ionization of helium atoms [2]. The other is the semiclassical model by Yudin and Ivanov [8]. In this model, they take into account the electron-core interaction, which modifies the tunneling position and the electron trajectory. This model explains the nonsequential double ionization of helium atoms in the intense laser field, quantitatively. Making use of the same model, Tong et al. $[9,10]$ studied the rescattering induced double ionization and dissociation of $\mathrm{H}_{2}$ and $\mathrm{D}_{2}$ molecules, and their results are also in good agreement with the experiments [3,11-13].

Unfortunately, the above two models treat the electron as a particle amenable to the classical dynamics and there is no phase information between the different trajectories in the simulations. We cannot use them to investigate the coherent dynamic processes, such as the momentum distribution of the low energy ATI [14] or molecular imaging $[6,7]$. The rescattering electron is only of a few percentage of the ionized wave packet, which in turn is a tiny portion of the total wave function. Thus it is difficult to extract the rescattering information by solving the time-dependent Schrödinger equation directly even though we can study the dynamic processes, like the momentum distribution of the low energy ATI [15]. The information cannot be obtained experimentally because the rescattering is an intermediate process and the experiments only observe the consequences of the rescattering. Recently, Smirnova et al. [16] suggested a pump-probe experiment to study the rescattering process. Even in their suggestions, one cannot obtain the information without the help of theoretical simulations. All these stimulate us to develop a new numerical scheme to obtain this information. Recently, we have developed a time-integral method [17] to study the carrier-envelope-phase dependent high energy ATI spectra and our results are in good agreement with the measurements [18]. We extend the method to study the rescattering process in this Letter.

The interaction of intense laser field with atoms or molecules can be described by a time-dependent Hamiltonian $H(t)$, which is the sum of the laser-fieldfree Hamiltonian $H_{0}$ and the laser-electron interaction 
$V(t)=-z E_{0} \cos (\omega t)$. Here we assume that the laser field is linearly polarized along the $z$ direction with the peak strength $E_{0}$ and the laser frequency $\omega$. We also assume that the laser pulse duration is infinity so that we can study the rescattering process without being entangled with the laser pulse duration. The dynamic processes of laser-atom interactions can be studied by the following integral equation (atomic units $m_{e}=\hbar=e=1$ are used)

$$
\Psi(t)=-i \int_{-\infty}^{t} e^{-i \int_{\tau}^{t} H\left(t^{\prime}\right) d t^{\prime}} V(\tau) e^{-i H_{0} \tau} \Phi_{0} d \tau+e^{-i H_{0} t} \Phi_{0},
$$

with $\Phi_{0}$ the initial wave function. The above integral equation is equivalent to the time-dependent Schrödinger equation under a proper initial condition. In Eq. (1), the first term on the right-hand side represents the initial wave function freely propagating to time $\tau$ in $H_{0}$, then interacting with the laser field at time $\tau$, and propagating further to time $t$ in the full Hamiltonian. The second term stands for $\Phi_{0}$ propagating to time $t$ in $H_{0}$ without interacting with the laser field. Defining

$$
\Psi_{j}(t)=-i \int_{t_{j-1}}^{t_{j}} e^{-i \int_{\tau}^{t} H\left(t^{\prime}\right) d t^{\prime}} V(\tau) e^{-i H_{0} \tau} \Phi_{0} d \tau,
$$

the time-dependent wave function $\Psi(t)$ is recast into

$$
\Psi(t)=\sum_{j} \Psi_{j}(t)+e^{-i H_{0} t} \Phi_{0} .
$$

$\Psi_{j}(t)$ corresponds to the electron interacting with the laser field directly for the first time in the interval from $t_{j-1}$ to $t_{j}$ and then propagating in the full Hamiltonian to time $t$. If we choose the time interval as a half-cycle period, namely, $\omega t_{j}=(2 j+1) \pi / 2$, the time-dependent wave function $\Psi(t)$ is the sum of each half-cycle's contribution. All the $\Psi_{j}(t)$ s are identical with a proper time shift so we only present $\Psi_{j}(t)$ with $j=0$. Taking hydrogen atoms in the intense laser fields as an example, we illustrate how to study the time evolution of the rescattering wave packet based on the above method. Of course, we can use this method to study other atoms or molecules under the single active electron approximation. In the present simulation, we choose the laser wavelength $800 \mathrm{~nm}$ and the laser intensity $2 \times 10^{14} \mathrm{~W} / \mathrm{cm}^{2}$ to cope with the rescattering induced experiments $[3,13]$.

Figure 1(a) shows a typical wave function immediately after the half-cycle ionization. We see that $\Psi_{j}(t)$ has two components in space. One represents the excitation in the inner region and the other stands for the tunneling ionization in the outer region. The excitation part will be ionized in the next half-cycle when the laser field changes its direction. To illustrate the rescattering process without the contamination of this indirect ionization, we remove the excitation component from $\Psi_{j}(t)$ when the "direct" ionization is over. Watching the time evolution of $\Psi_{j}(t)$ [19], we notice that the electron wave packet moves back and forth and goes out with the structures similar to the momentum distribution of low energy ATI spectra [14]. However, we cannot see the rescattering wave packet clearly from $\Psi_{j}(t)$. The visualization of $\Psi_{j}(t)$ does not provide the detailed information explicitly. Our goal is to study the rescattering wave packet quantitatively, namely, the time-dependent momentum distribution of the rescattering wave packet.

For such a purpose, we have to find a way to present the rescattering wave packet intuitively and quantitatively. Let us look at what we did in the semiclassical model to get the time-dependent energy distributions. The electron is first ionized through the tunneling ionization when the laser field reaches its peak strength. The classical trajectory of this ionized electron is calculated. If the trajectory is close to the parent core at time $t_{r}$, we regard this event as the rescattering electron incident from the asymptotic region in the absence of the laser field. We analyze $\Psi_{j}(t)$ in a similar way. First, we calculate the laser-field-free continuum wave function $\psi_{l}(E, r)$ for a given energy $E$ and a partial
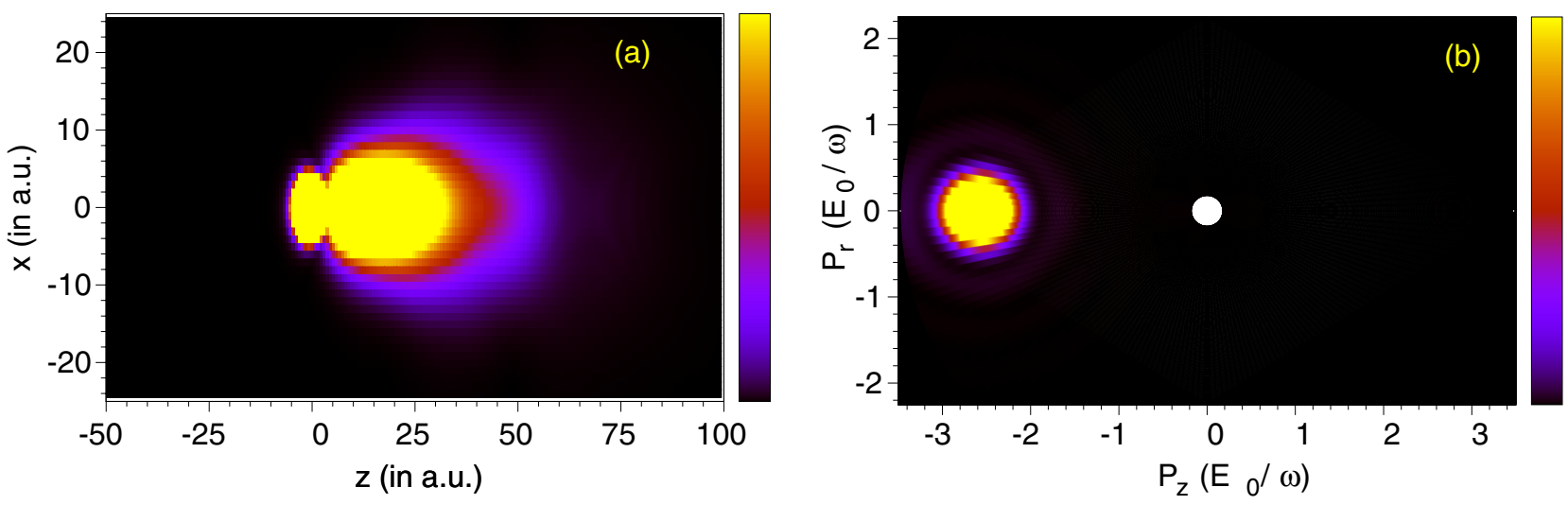

FIG. 1 (color online). (a) The wave function $\left|\Psi_{j}(t)\right|^{2}$ at $t=\pi /(2 \omega)$ and (b) momentum distribution $|M(E, \Omega, t)|^{2}$ at the first returning time. 
wave $l$ solving the Schrödinger equation numerically as

$$
H_{0} \psi_{l}(E, r)=E \psi_{l}(E, r),
$$

with imposing $r \psi_{l}(E, r) \rightarrow 0$ as $r \rightarrow 0$, and

$$
\lim _{r \rightarrow \infty} r \psi_{l}(E, r)=\sqrt{\frac{2}{\pi k}} \sin \left(k r+\frac{1}{k} \ln (2 k r)-\frac{l \pi}{2}+\delta_{l}(E)\right) .
$$

Here $k=\sqrt{2 E}$ and $\delta_{l}(E)$ is the phase shift. We project $\Psi_{j}(t)$ onto $\left\{\psi_{l}(E, r)\right\}$ states in the inner region when the wave packet returns to the parent core as

$$
C_{l}(E, t)=\int f\left(r, R_{c}\right) Y_{l 0}^{*}(\hat{\mathbf{r}}) \psi_{l}^{*}(E, r) \Psi_{j}(t) d \mathbf{r} .
$$

$f\left(r, R_{c}\right)$ is introduced to define the size of the inner-region $R_{c}$ with the boundary width $\Delta$ as

$$
f\left(r, R_{c}\right)=\left\{\begin{array}{ll}
1.0 & \text { for } r<R_{c} \\
e^{-\left(r-R_{c}\right)^{2} / \Delta^{2}} & \text { for } r \geq R_{c}
\end{array} .\right.
$$

In the present calculation, $R_{c}$ is 5 to 10 a.u. and $\Delta$ is 2 a.u. The results are not sensitive to the choice of $R_{c}$ and $\Delta$. With $C_{l}(E, t)$ and $\delta_{l}(E)$, we separate the wave packets into an incoming spherical wave $\exp (-i k r) / r$ and an outgoing spherical wave $\exp (i k r) / r$ in the asymptotic region. The rescattering wave packet is of the form of the incoming spherical wave. Defining

$$
M(E, \Omega, t)=\sum_{l} i^{l} C_{l}(E, t) e^{-i \delta_{l}(E)} Y_{l m}(\Omega),
$$

the wave function of the rescattering electron in the asymptotic region with incident energy $E$ from incident direction $\Omega$ is $M(E, \Omega, t) \exp (-i k r) / r$. The rescattering momentum distributions at time $t$ is given by

$$
\frac{d P(E, \Omega, t)}{d E d \Omega}=|M(E, \Omega, t)|^{2} .
$$

$M(E, \Omega, t)$ contains all the dynamic information of the rescattering wave packet including the phase. If we can measure the dynamics due to the recollision of this wave packet with the core, we can interrogate the core structure with the help of calculated $M(E, \Omega, t)$. Note that adding the contribution of $\Phi_{0}$ to $\Psi_{j}$ in Eq. (5) does not change the results due to the orthogonality between $\Phi_{0}$ and $\psi_{l}(E, r)$. We also tried to project the $\Psi_{j}(t)$ onto the plane waves and the results are in agreement with the present results only in the high energy region.

Are the simulated results consistent with the intuitive physical picture of the rescattering theory? Figure 1(b) shows the rescattering momentum distribution at the first returning time. It does show that the electron is bounced back by the laser field from the right side and revisits the core with the rescattering energy around $4 U_{p}$ with $U_{p}=$ $E_{0}^{2} /\left(4 \omega^{2}\right)$, higher than the classical one. The timedependent momentum distribution [20] shows that the electron moves back and forth in the momentum space. We notice that the rescattering momentum from the right side, which corresponds to the odd number returns, is higher than that from the left side, which corresponds to the even number returns. All these are consistent with the classical predictions and we do reproduce the intuitive classical pictures of the rescattering theory.

Next, we compare the percentages of the electron wave packet coming back to the parent core at time $t$ with energy $E$ calculated by the quantum and semiclassical methods. The associated electron spectra are defined as

$$
\frac{d \bar{P}(E, t)}{d E}=\frac{100}{A_{i}} \int \frac{d P(E, \Omega, t)}{d E d \Omega} d \Omega,
$$

with $A_{i}$ the ionization probability in the half-cycle period calculated by the corresponding method. Figure 2 shows the energy and returning time distributions of the quantum and semiclassical calculations. First, we see that only a few percent of the ionized wave packet revisits the parent-core. Our calculated returning times are in good agreement with the classical and semiclassical ones and the returning time is model insensitive. Nevertheless, we observe a sharp contrast between the electron energy distribution by the quantum and semiclassical methods at the returning times
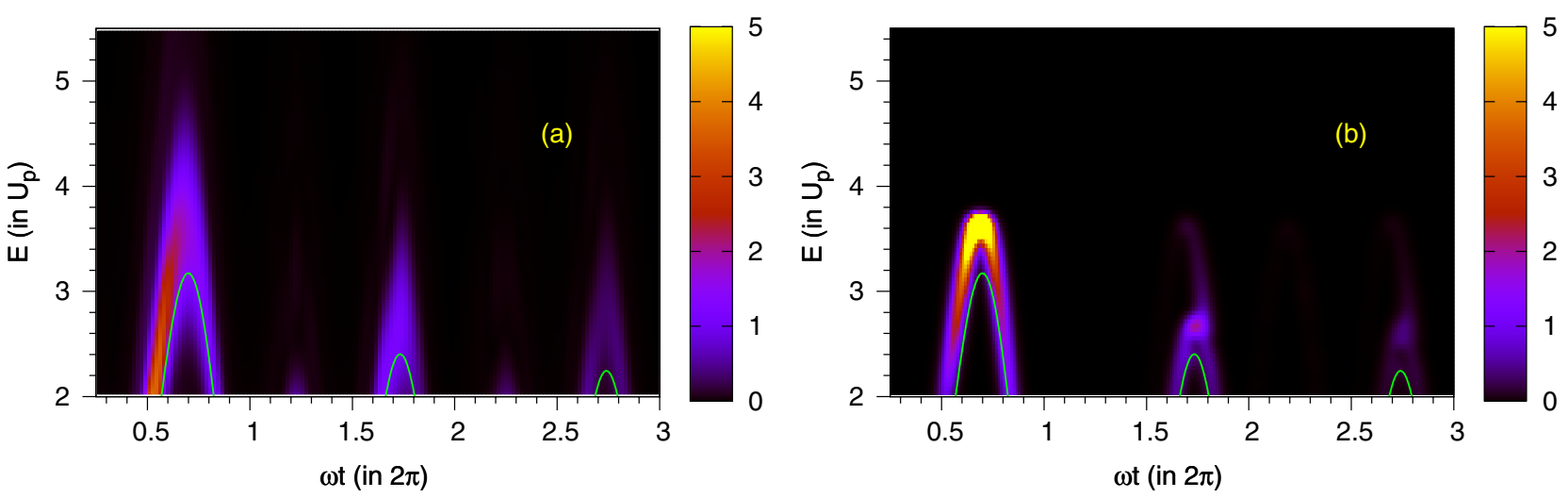

FIG. 2 (color online). The rescattering energy and time distributions from (a) the quantum calculation and (b) the semiclassical calculation. The solid curves are from the classical model. The color coding represents the value of $\frac{d \bar{P}(E, t)}{d E}$. 


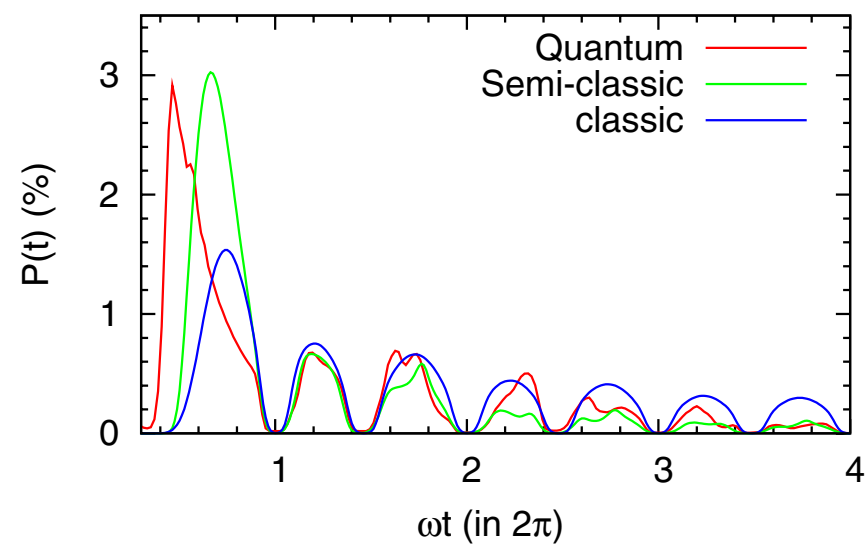

FIG. 3 (color online). The percentage of the rescattering wave packet as a function of time $t$.

as shown in Fig. 2. The quantum simulation shows the striking feature that the rescattering electron can distribute over the higher-energy region $\left(E>4 U_{p}\right)$ compared with the results by the other two simulations. The returning energy by the quantum calculations is even larger than $5 U_{p}$ if the laser intensity decreases to $1 \times 10^{14} \mathrm{~W} / \mathrm{cm}^{2}$. It is speculated that this phenomenon would be ascribable to the quantum coherence of many electron trajectories, namely, the phase effect ignored in the other two simulations. This finding is of great importance for proper evaluations and understandings of some rescattering induced processes, which mostly coincides with the energy region $\left(E>4 U_{p}\right)$ pointed out above for the intensity $1 \times$ $10^{14} \mathrm{~W} / \mathrm{cm}^{2}$. For instance, the rescattering induced dissociation process [3] is responsible for the rescattering electron energy near the margin of the excitation energy. The high energy rescattering electron, which is missed in the semiclassical results, also responds to the generation of attosecond pulse [4] near the cutoff region. Therefore, it is convinced that the reliable rescattering energy distribution is highly desired to decide if these processes are possible or not.

Finally, to compare with the classical predictions, we define a time-dependent rescattering probability

$$
P(t)=\int \frac{d \bar{P}(E, t)}{d E} d E .
$$

Figure 3 shows $P(t)$ calculated by the three methods. As we see, the first returning times of the quantum and semiclassical calculations are earlier than that of the classical model. This is because many low energy electrons contribute to the first return as shown in Fig. 2. The low energy returning electrons do not contribute to the later returns so that the later returning times agree with one another. The probabilities of the second and third returns do not differ much among the three methods. For the later returns, the quantum and semiclassical results decrease faster than that of the classical model. This is because the electrons are lost during the recollision with the parent core and the effect is completely ignored in the classical model.

To summarize, we have reproduced the rescattering wave packet in the intense laser-atom interaction by a full-quantum, nonperturbative method. The motion of the rescattering wave packet is consistent with the classical trajectory qualitatively. However, the quantum description of the rescattering is found very important to show the large fraction of the electrons with the higher energies than those evaluated by the semiclassical and classical descriptions. These rescattering electrons are speculated to play significant roles in some rescattering induced processes. The present method can also be used to study atoms or molecules in the ultrashort pulsed laser field. Shaping the ultrashort laser field, we can manipulate the momentum distribution of the rescattering electron, consequently, control the attosecond dynamics.

This work was supported by Grant-in-Aids for Scientific Research (B) and (C) from the Japan Society for the Promotion of Science.

*tong@ims.tsukuba.ac.jp

[1] P. B. Corkum, Phys. Rev. Lett. 71, 1994 (1993).

[2] B. Walker et al., Phys. Rev. Lett. 73, 1227 (1994).

[3] M.F. Kling et al., Science 312, 246 (2006).

[4] A. Baltuska et al., Nature (London) 421, 611 (2003).

[5] P. Agostini and L. F. DiMauro, Rep. Prog. Phys. 67, 813 (2004).

[6] J. Itatani et al., Nature (London) 432, 867 (2004).

[7] S. X. Hu and L. A. Collins, Phys. Rev. Lett. 94, 073004 (2005).

[8] G. L. Yudin and M. Y. Ivanov, Phys. Rev. A 63, 033404 (2001).

[9] X. M. Tong, Z. X. Zhao, and C. D. Lin, Phys. Rev. A 68, 043412 (2003).

[10] X. M. Tong and C. D. Lin, Phys. Rev. Lett. 98, 123002 (2007).

[11] H. Niikura et al., Nature (London) 417, 917 (2002).

[12] A. S. Alnaser et al., Phys. Rev. Lett. 91, 163002 (2003).

[13] A. S. Alnaser et al., Phys. Rev. Lett. 93, 183202 (2004).

[14] C. M. Maharjan, A. S. Alnaser, I. Litvinyuk, P. Ranitovic, and C. L. Cocke, J. Phys. B 39, 1955 (2006).

[15] M. Wickenhauser, X. M. Tong, and C. D. Lin, Phys. Rev. A 73, 011401(R) (2006).

[16] O. Smirnova, S. Patchkovskii, and M. Spanner, Phys. Rev. Lett. 98, 123001 (2007).

[17] X. M. Tong, K. Hino, and N. Toshima, Phys. Rev. A 74, 031405(R) (2006).

[18] G. G. Paulus et al., Phys. Rev. Lett. 91, 253004 (2003).

[19] See EPAPS Document No. E-PRLTAO-99-048735 for the time-dependent wave function in space. For more information on EPAPS, see http://www.aip.org/pubservs/ epaps.html.

[20] See EPAPS Document No. E-PRLTAO-99-048735 for the time-dependent momentum distribution of the rescattering wave packet. For more information on EPAPS, see http:// www.aip.org/pubservs/epaps.html. 